\title{
Nueva especie de Loasa (Loasaceae) de Chile
}

\section{A new species of Loasa (Loasaceae) from Chile}

\author{
Mélica Muñoz-Schick ${ }^{1}$ \& Alain de Trenqualye ${ }^{2}$ \\ ${ }^{1}$ Museo Nacional de Historia Natural, Casilla 787, Santiago, Chile. \\ ${ }^{2}$ Luis Pasteur 6006, Depto. 302. Santiago, Chile \\ mmunoz@mnhn.cl; atrenqua@gmail.com
}

\begin{abstract}
Loasa mollensis Muñoz-Schick et Trenqualye is described as a new species. It is apparently narrowly endemic to a small area in the coast of the Valparaiso region, central Chile. The species has small yellow flowers and infer rounded capsules containing large seeds.
\end{abstract}

El género Loasa es uno de los más diversos de la flora de Chile: diferentes tratamientos le asignan una riqueza de entre 45 y 31 especies, de las cuales al menos 18 serían endémicas del país (Marticorena 1990, Weigend et al. 2004, Zuloaga et al. 2008, Moreira-Muñoz 2011). Tal como sucede en otros géneros diversos de la flora de Chile, como Alstroemeria (e.g. Muñoz-Schick et al. 2011), es esperable que se sigan descubriendo nuevas especies o que haya que recurrir a nuevos tratamientos nomenclaturales para clarificar las especies de Loasa. En este caso, se trata de una especie de Loasa que ha pasado desapercibida posiblemente debido a sus flores pequeñas, poco llamativas.

En diversas visitas al sector de Los Molles, V Región de Valparaíso, fuimos alertados de una especie de Loasa que no aparecía en la bibliografía correspondiente. Se recolectó y fotografió material, comparando con la literatura (Gay 1846, Urban 1900, Reiche 1901,1902, Weigend et al. 2004), y ejemplares de Herbario presentes en SGO y en CONC y no se encontró dicha especie. Los trabajos específicos del área no mencionan la especie: Mooney y Schlegel (1967) no tratan las especies herbáceas, mientras que Lund (2002) menciona dos especies de Loasa: L. tricolor Ker.-G. y una Loasa sp., que podría ser la especie aquí descrita.

\section{DESCRIPCIÓN DE LA ESPECIE}

Loasa mollensis Muñoz-Schick et Trenqualye, sp. nov. TIPO: Chile. Región de Valparaíso, Los Molles, lado derecho después del portón de entrada al Parque privado Los Molles, 32¹4'20,7' S; 71³1'4,1” W, 30 m s.n.m., M. Muñoz 5130, 10-X-2010, SGO 160201 (Holotipo), SGO 160202 (Isotipo).

Caule volubilis, folia opposita, petiolata, triloba, lobis rotundatis, medio productiore subdentatis, 1-flori, axillaris et apicalis, parvis, sepala lanceolatis, petala ovato-cuculatis, lutea, squamulae florales extus 3-appendiculate, apendices filiformibus cum apice dilatata, capsula rotundata.

Planta anual, de tallo voluble que puede sobrepasar los $4 \mathrm{~m}$ de longitud apoyándose sobre arbustos, con pelos urticantes débiles; hojas opuestas, con el pecíolo más corto que la lámina, ésta trilobulada, con 5 nervios que parten desde su base, de 3-4 (-5) cm de largo por 3,6 (-7) cm de ancho en los lóbulos inferiores, éstos algo más cortos que el superior, y a veces tienen un diente o casi un lóbulo en la parte inferior, lo mismo que el lóbulo superior que tiene un diente a cada lado en la parte media; flores terminales, pequeñas, de 7-10 mm de diámetro, generalmente solitarias en la axila de las hojas y en el ápice del tallo, con pedicelos de 7- 20 (-30) mm de largo; 5 sépalos abiertos, lanceolados, de 2-4 mm de largo, con 3 estrías longitudinales, poco más cortos que los 5 pétalos amarillos, cuculiformes, adelgazados hacia la base. Escamas nectaríferas de $2 \mathrm{~mm}$ de largo, verde amarillentas con una franja roja poco más arriba del centro, otra más difusa hacia la base, que se hacen más notorias con la madurez de la flor, y terminadas en dos lóbulos redondeados con un notorio borde blanco; poseen 3 apéndices o filamentos dorsales que nacen cerca de la base, de color verde la mitad inferior, con una línea roja en el centro, blanquecinos y ensanchados como porra hacia el ápice, estos apéndices varían de largo (a medida de la madurez de la flor) desde más cortos que las escamas a más largos que ellas; numerosos estambres de filamentos blancos casi el doble de largo de las escamas. Cápsula ínfera, de base redondeada, de 1,5-2 cm de diámetro, mostrando cierta transparencia en la parte superior. Contiene ca. 6 
semillas oblongas, reticuladas, de color café, de $4 \mathrm{~mm}$ de largo (Fig. 1).

\section{PARATIPO:}

CHILE. Región de Valparaíso, Los Molles, después del portón de entrada al Parque privado Los Molles, A. de Trenqualye, 23-IX-2010, SGO 160474.

Endémica de la Región de Valparaíso, se encuentra en sendero de inicio del sitio Los Molles; se observó también en la falda oriental de un montículo con conchales en el sendero que va hacia el Puquén; en general apoyándose en plantas arbustivas achaparradas de litre (Lithrea caustica (Molina) Hook. et Arn.). Florece desde agosto hasta octubre.

Luebert y Pliscoff (2006) denominan a esta unidad de
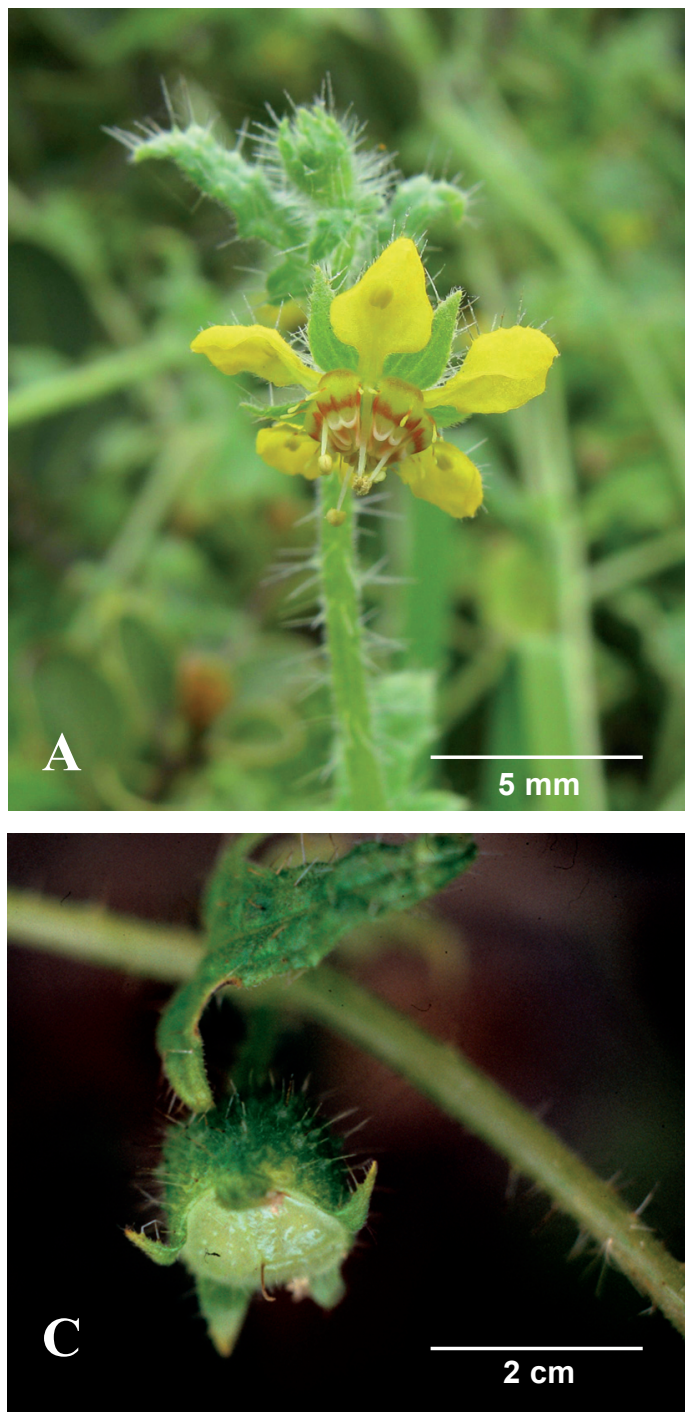

vegetación "Matorral Arborescente Esclerófilo Mediterráneo Costero de Peumus boldus y Schinus latifolius", con presencia además de Pouteria splendens (A. DC.) Kuntze, "lúcumo" asociado a roqueríos costeros.

Con sus pocas y grandes semillas, la nueva especie podría pertenecer al grupo Loasa serie Macrospermae Urb. \& Gilg., pero la mayoría de las especies de este grupo son plantas erguidas con hojas grandes, quinquelobadas, flores grandes y los apéndices de las escamas son dilatados en la base y terminados en un filamento, lo que no concuerda con nuestra especie. Con Loasa micrantha Poepp., de la Serie Volubles, comparten el pequeño tamaño de sus flores, las escamas y la forma de los apéndices, pero aquélla tiene flores largamente pedunculadas, de pétalos blancos y semillas pequeñas.

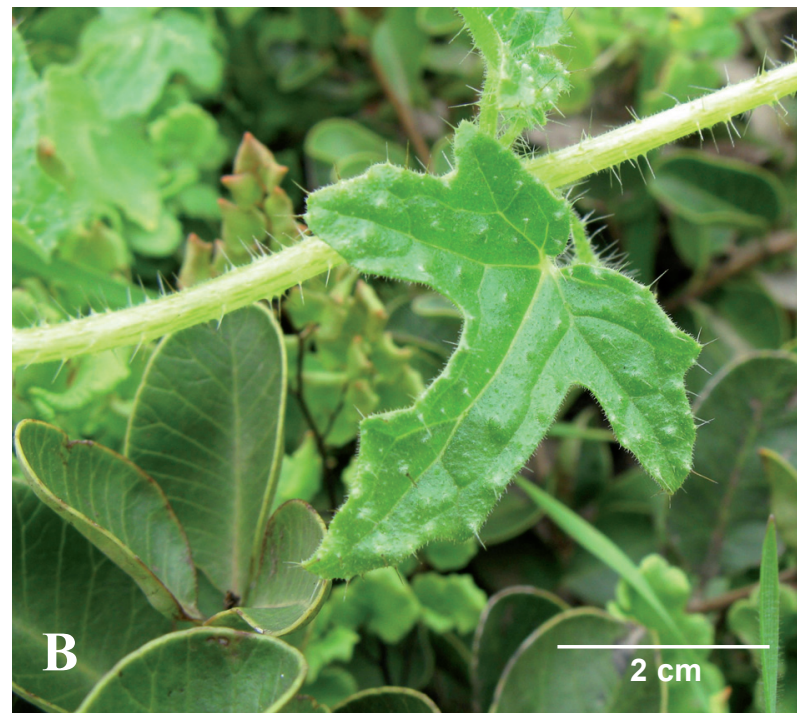

D
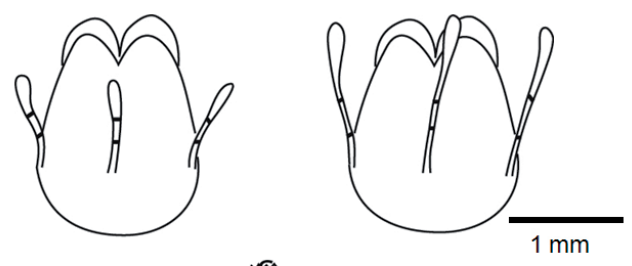

11

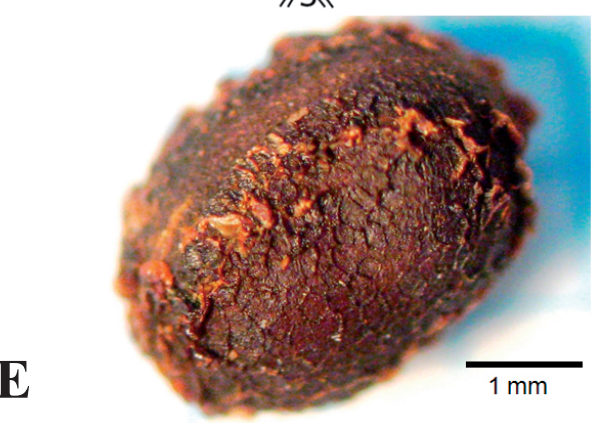

Figura 1. Loasa mollensis. A. Detalle de flor. B. Hoja media. C. Fruto. D. Detalle de escamas y sus apéndices E. Semilla (Fotografías: A-B Alain de Trenqualye. C. Sergio Moreira . D. Original M. Muñoz. E. Víctor Ardiles).

FIGURE 1. Loasa mollensis. A. Flower detail. B. Medial leaf. C. Fruit. D. Scales and appendages details. E. Seed (Photographs: A-B Alain de Trenqualye, C. Sergio Moreira. D. Original M. Muñoz. E. Víctor Ardiles). 
COMENTARIO FINAL

Se presenta un cuadro sinóptico de las especies presentes en Chile y países limítrofes y su distribución ordenada de norte a sur (Zuloaga et al. 2008). En la Tabla 1 se observa que la Región de Coquimbo es la que presenta mayor cantidad de especies (18).

La especie estudiada permanecía hasta ahora desconocida, posiblemente debido a lo poco llamativa de sus flores pequeñas.

En cuanto a su conservación, estaría relativamente protegida en el parque privado Los Molles. El lugar ha sido muy visitado y apreciado por los botánicos debido a la presencia de especies como Pouteria splendens (A. DC.)
Kuntze, Puya venusta Phil. y Alstroemeria pelegrina L. Por ello ya en el año 1996 fue declarado como Sitio Prioritario para la Conservación de Biodiversidad de Chile (Muñoz et al. 1996). Ello sería recogido por la Estrategia Regional para la Conservación de la Diversidad Biológica (CONAMAPNUD 2005), que califica al sitio Los Molles en primera prioridad a nivel regional. Felizmente los dueños del predio han decidido crear un parque privado que en este momento tiene bajo protección esta notable comunidad florística. Loasa mollensis se suma ahora a esta lista de especies muy importantes de conservar y de conocer por parte de los visitantes que accedan a este santuario natural.

TABLa I. Especies del género Loasa en Chile y su distribución de norte a sur (*)

TABLE I. North to South distribution of Loasa genus species across Chile.

\begin{tabular}{|c|c|c|c|c|c|c|c|c|c|c|c|c|c|c|c|}
\hline \multirow[t]{2}{*}{ Loasa } & \multirow[t]{2}{*}{ Perú } & \multirow[t]{2}{*}{ Argentina } & \multicolumn{13}{|c|}{ ChILE } \\
\hline & & & I & II & III & IV & $\mathrm{V}$ & $\mathrm{M}$ & VI & VII & VIII & IX & $\mathrm{X}$ & $\mathrm{XI}$ & XII \\
\hline nitida & $\mathrm{x}$ & & $\mathrm{x}$ & $\mathrm{x}$ & & & & & & & & & & & \\
\hline longiseta & & & $\mathrm{x}$ & $\mathrm{x}$ & $\mathrm{x}$ & $\mathrm{x}$ & & & & & & & & & \\
\hline malesherbioides & & $\mathrm{x}$ & & $\mathrm{x}$ & $\mathrm{x}$ & & & & & & & & & & \\
\hline elongata & & & & $\mathrm{x}$ & $\mathrm{x}$ & $\mathrm{x}$ & & & & & & & & & \\
\hline multifida & & & & & $\mathrm{x}$ & $\mathrm{x}$ & & & & & & & & & \\
\hline caespitosa & & & & & $\mathrm{x}$ & $\mathrm{x}$ & $\mathrm{x}$ & $\mathrm{x}$ & & & & & & & \\
\hline pallida & & & & & $\mathrm{x}$ & $\mathrm{x}$ & $\mathrm{x}$ & $\mathrm{x}$ & & & & & & & \\
\hline tricolor & & $\mathrm{x}$ & & & $\mathrm{x}$ & $\mathrm{x}$ & $\mathrm{x}$ & $\mathrm{x}$ & & & & & & & \\
\hline placei & & & & & $\mathrm{x}$ & $\mathrm{x}$ & $\mathrm{x}$ & $\mathrm{x}$ & $\mathrm{x}$ & & & & & & \\
\hline sigmoidea & & $\mathrm{x}$ & & & $\mathrm{x}$ & $\mathrm{x}$ & & $\mathrm{x}$ & & $\mathrm{x}$ & & & & & \\
\hline sclareifolia & & $\mathrm{x}$ & & & $\mathrm{x}$ & $\mathrm{x}$ & $\mathrm{x}$ & $\mathrm{x}$ & $\mathrm{x}$ & $\mathrm{x}$ & $\mathrm{x}$ & $\mathrm{x}$ & & & \\
\hline triloba & & & & & $\mathrm{x}$ & $\mathrm{x}$ & $\mathrm{x}$ & $\mathrm{x}$ & $\mathrm{x}$ & $\mathrm{x}$ & $\mathrm{x}$ & $\mathrm{x}$ & & & \\
\hline arnottiana & & & & & & $\mathrm{x}$ & & & & & & & & & \\
\hline illapelina & & & & & $\mathrm{x}$ & $\mathrm{x}$ & & & & & & & & & \\
\hline floribunda & & & & & & $\mathrm{x}$ & $\mathrm{x}$ & $\mathrm{x}$ & & & & & & & \\
\hline prostrata & & & & & & $\mathrm{x}$ & $\mathrm{x}$ & $\mathrm{x}$ & $\mathrm{x}$ & & & & & & \\
\hline heterophylla & & $\mathrm{x}$ & & & & $\mathrm{x}$ & $\mathrm{x}$ & $\mathrm{x}$ & & $\mathrm{x}$ & & & & & \\
\hline insons & & $\mathrm{x}$ & & & & $\mathrm{x}$ & $\mathrm{x}$ & $\mathrm{x}$ & $\mathrm{x}$ & $\mathrm{x}$ & & & & & \\
\hline lateritia & & $\mathrm{x}$ & & & & $\mathrm{x}$ & & & & $\mathrm{x}$ & $\mathrm{x}$ & $\mathrm{x}$ & & & \\
\hline acerifolia & & $\mathrm{x}$ & & & & $\mathrm{x}$ & & & & $\mathrm{x}$ & $\mathrm{x}$ & $\mathrm{x}$ & $\mathrm{x}$ & & \\
\hline mollensis & & & & & & & $\mathrm{x}$ & & & & & & & & \\
\hline paradoxa & & & & & & & $\mathrm{x}$ & $\mathrm{x}$ & & & & & & & \\
\hline volubilis & & & & & & & $\mathrm{x}$ & $\mathrm{x}$ & & $\mathrm{x}$ & $\mathrm{x}$ & $\mathrm{x}$ & & & \\
\hline sagittata & & & & & & & $\mathrm{x}$ & & & & & & $\mathrm{x}$ & & \\
\hline micrantha & & & & & & & & $\mathrm{x}$ & $\mathrm{x}$ & $\mathrm{x}$ & $\mathrm{x}$ & $\mathrm{x}$ & & & \\
\hline pinnatifida & & $\mathrm{x}$ & & & & & & $\mathrm{x}$ & $\mathrm{x}$ & $\mathrm{x}$ & $\mathrm{x}$ & $\mathrm{x}$ & $\mathrm{x}$ & & \\
\hline humilis & & & & & & & & & & $\mathrm{x}$ & & & & & \\
\hline acanthifolia & & $\mathrm{x}$ & & & & & & & & $\mathrm{x}$ & $\mathrm{x}$ & $\mathrm{x}$ & $\mathrm{x}$ & & \\
\hline gayana & & & & & & & & & & & & $\mathrm{x}$ & $\mathrm{x}$ & & \\
\hline martinii & & & & & & & & & & & & $\mathrm{x}$ & $\mathrm{x}$ & & \\
\hline nana & & $\mathrm{x}$ & & & & & & & & & & $\mathrm{x}$ & $\mathrm{x}$ & & \\
\hline argentina & & $\mathrm{x}$ & & & & & & & & & & & & $\mathrm{x}$ & $\mathrm{x}$ \\
\hline bergii & & $\mathrm{x}$ & & & & & & & & & & & & $\mathrm{x}$ & $\mathrm{x}$ \\
\hline
\end{tabular}

(*) Basado en Zuloaga et al. (2008) con modificaciones./ Based on Zuloaga et al. (2008) with modifications. 


\section{AGRADECIMIENTOS}

Se agradece al químico-farmacéutico Sr. Claudio Silva, quien nos alertó de su existencia. A Sergio Moreira por la foto del fruto. A Víctor Ardiles por la foto de la semilla. A Alicia Marticorena por el préstamo de materiales de Loasa desde CONC.

\section{BIBLIOGRAFÍA}

CONAMA-PNUD. 2005. Estrategia y Plan de Acción para la Conservación de la Diversidad Biológica, Región de Valparaíso. 225 pp.

GaY, C. 1846. Loasáceas, En: Historia Física y Política de Chile. Tomo 2: 367-401.

Lund, R. 2002. Flora del cabo de Los Molles (Chile, Región de Valparaíso). Estudio monográfico para optar al Grado Académico de Licenciatura en Ciencias y Artes Ambientales, Escuela de Ecología y Paisajismo, Facultad de Arquitectura y Bellas Artes de la Universidad Central. 61 pp., más Apéndices.

Luebert, F. \& P. Pliscoff. 2006. Sinopsis bioclimática y vegetacional de Chile. Editorial Universitaria, 316 pp.

Marticorena, C. 1990. Contribución a la estadística de la flora vascular de Chile. Gayana Botánica 47(3-4): 85-113.

Mooney, H.A. \& F. Schlegel. 1967. La vegetación costera del cabo de Los Molles en la provincial de Aconcagua. Boletín Universidad de Chile 75: 27-32.
Moreira-Muñoz, A. 2011. Plant Geography of Chile. Plant and Vegetation, vol.5, Springer $343 \mathrm{pp}$

MuÑoz, M. 1981. Comentario sobre la distribución de Loasa sagittata Hook. et Arn. Noticiario Mensual, Museo Nacional de Historia Natural (Chile) 297: 14.

MuÑoz, M., H. NúÑEZ \& J. YÁnEZ. (eds.) 1996. Libro Rojo de los Sitios Prioritarios para la Conservación de la Diversidad Biológica de Chile. Corporación Nacional Forestal, Ministerio de Agricultura. 203 pp.

Muñoz-Schick, M., V. Morales \& A. Moreira-MuÑoz 2011. Validación de Alstroemeria parvula Phil. (Alstroemeriaceae). Gayana Botánica 68(1): 110-112.

Reiche, C. 1901. Loasaceae. Estudios críticos sobre la flora de Chile. Anales Universidad de Chile 108: 707-751.

Reiche, C. 1902. Loasaceae. Flora de Chile, Tomo 3: 211-255.

Urban, I. 1900. Monographia Loasacearum. Nov. Acta Abh. der Kaiserl. Leop. Carol. Deutschen Akad. Natur., Halle, 76(1): 1-384, 8 lám.

Weigend, M., M. Gottschling, S. Hoot \& M. Ackermann. 2004. A preliminary phylogeny of Loasaceae subfam. Loasoideae (Angiospermae: Cornales) based on trnL (UAA) sequence data, with consequences for systematics and historical biogeography. Organisms, Diversity \& Evolution 4: 7390.

Zuloaga, F.O., O. Morrone \& M.J. Belgrano (eds.). 2008. Catálogo de las Plantas Vasculares del Cono Sur (Argentina, Sur de Brasil, Chile, Paraguay y Uruguay). Monographs of the Missouri Botanical Garden 107, vol. 3, Dicotyledoneae: Fabaceae (Senna-Zygia) Zygophyllaceae. 2287-3348

Recibido: 12.03 .11

Aceptado: 05.08.11 